\section{Porines \\ bactériennes \\ et sensibilité \\ aux antibiotiques}

Jean-Marie Pagès bactériennes sont une des voies principales d'entrée pour les antibiotiques usuels comme les $\beta$-lactamines et les fluoroquinolones. L'expression de ces protéines, ainsi que les interactions et les acides aminés qui organisent la diffusion dans le pore sont des éléments majeurs de la perméabilité membranaire. Ainsi, plusieurs isolats cliniques de bactéries résistantes présentant des défauts de porines ou exprimant des porines modifiées ont été décrits. Les caractérisations de divers répresseurs ou activateurs de la synthèse des porines, mais aussi de molécules pouvant moduler leur activité «canal», ont montré la complexité et la flexibilité des facteurs contrôlant l'expression fonctionnelle de ces protéines. Cet article illustre notamment comment la régulation de l'expression des porines et les mécanismes qui organisent la diffusion des solutés peuvent être des paramètres-clés de la résistance bactérienne aux antibiotiques. <

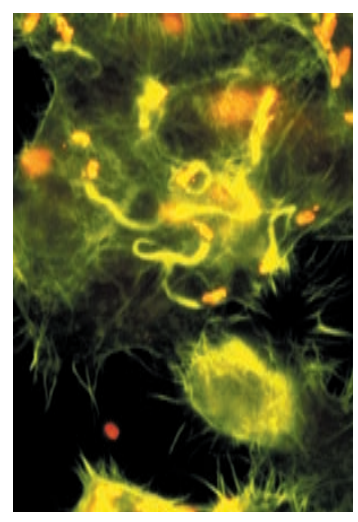

EA2197, Enveloppe bactérienne, perméabilité et antibiotiques, 27, boulevard Jean Moulin, 13385 Marseille Cedex 05, France.

\section{Lean-Marie.PAGES@} medecine.univ-mrs.fr

cet article, permettent de mieux appréhender la relation entre les porines, la perméabilité membranaire et la résistance aux antibiotiques.

\section{Membrane externe et porine}

\section{Membrane externe}

La membrane externe des bactéries à Gram négatif forme une barrière efficace qui protège la machinerie cellulaire contre les agents toxiques externes: agressions physiques (choc osmotique, température), chimiques (détergents, acides), biologiques (défensines, bactériocines) ou autres (antibiotiques, anticorps) (Figure 1). Le contrôle de la perméabilité et de l'intégrité membranaires joue un rôle primordial dans cette défense naturelle de la bactérie. La pénétration des molécules chargées est affectée par la couche de LPS (et de phospholipides). La densité des sucres et des chaînes latérales du LPS organise un filtre efficace pour les autres molécules [1-3].

Dans les bactéries à Gram négatif, il existe des canaux membranaires qui assurent la diffusion de diverses classes de molécules à travers la membrane externe. Ces systèmes de transport passif assurent la pénétration des constituants indispensables à la vie bactérienne tels que les sucres, les acides aminés ou les sels. 


\section{Perméabilité membranaire et porines}

Trois classes principales de protéines intervenant dans la perméabilité de la membrane externe ont été identifiées [4]: les porines non spécifiques, telles que $0 \mathrm{mpF}$, $0 \mathrm{mpC}$, PhoE, $0 \mathrm{mpD}$ et $0 \mathrm{mp} 36$; les porines sélectives impliquées dans la diffusion de sucres ou de métaux, comme LamB ou FhuA; les protéines, telles que $0 \mathrm{mpA}$ et TolC, formant des canaux pour diverses molécules.

Les activités de canal membranaire associées à ces protéines ont été caractérisées par différentes méthodes [11-24]: cinétiques d'accumulation de composés radiomarqués ou fluorescents, mesures de diffusion dans des liposomes contenant la porine purifiée, mesures de physicochimie déterminant la conductance, la sélectivité, et la sensibilité au potentiel des canaux après reconstitution de la protéine dans des bicouches lipidiques ou dans des systèmes de patch clamp, et expériences de compétition utilisant des produits bloquant le canal.

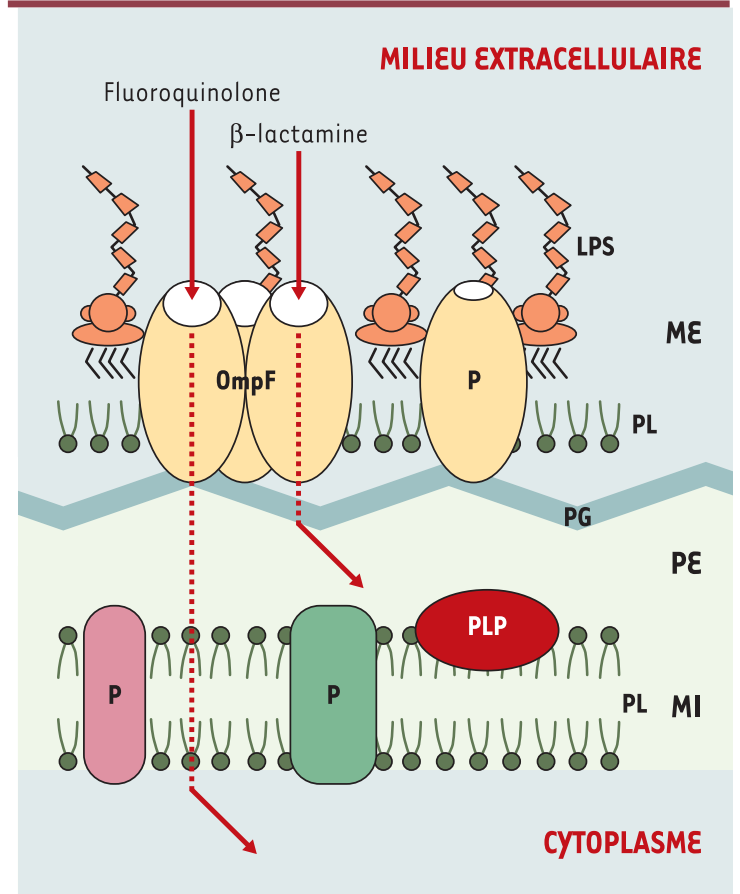

Figure 1. Enveloppe d'Escherichia coli. L'enveloppe des bactéries à Gram négatif comprend deux membranes: la membrane interne (MI) et la membrane externe (ME) séparées par le périplasme $(P E)$. La membrane interne est un double feuillet phospholipidique contenant diverses protéines (transporteurs, enzymes...). La membrane externe est une bicouche asymétrique constituée du lipopolysaccharide (LPS) et de phospholipides (PL) où s'insèrent de nombreuses protéines. Cette représentation simplifie la barrière que constitue la membrane externe pour les molécules hydrophiles (ici, $\beta$-lactamine ou fluoroquinolone) qui utilisent la porine $0 \mathrm{mpF}(0 \mathrm{mpF})$. P: protéines; PG: peptidoglycane; PLP: protéine de liaison aux pénicillines.
La fonction canal a été ainsi rapportée pour plusieurs protéines majeures de la membrane externe: $0 \mathrm{mpF}$, $0 \mathrm{mpC}, 0 \mathrm{mpD}$, Phoz, LamB, $0 \mathrm{mpA}, 0 \mathrm{mpK} 36$ et $0 \mathrm{mp} 36$. Le terme générique de porine, attribué historiquement à $0 \mathrm{mpF}$ et $0 \mathrm{mpC} \mathrm{d}^{\prime} \varepsilon$. coli, a été étendu aux protéines homologues en séquence et en activité, comme 0mpK36, 0mpD, 0mp36 de Klebsiella pneumoniae, Enterobacter aerogenes, Salmonella typhimurium. Par ailleurs, la structure tridimensionelle a été résolue pour certaines de ces porines (Tableau I, Figure 2) [4-10], et des analyses structure-fonction ont été réalisées.

\section{Boucle L3 et diffusion}

Le pore $0 \mathrm{mpF}$, comme ceux formés par $0 \mathrm{mpC}, 0 \mathrm{mpK} 36$ et PhoE, montre une distribution particulière des charges, due aux acides aminés dans la région de constriction interne du canal formée par le repliement de la boucle L3 (Figures 2 et 3). Un croissant de charges négatives (Asp et Glu) opposé à une face de charges positives (Arg et Lys) induit un champ électrostatique [26]. Cette région est fortement conservée dans les porines des entérobactéries [27]. Outre des contraintes stériques induites par la région de constriction, le champ électrostatique impose des contraintes d'orientation aux solutés chargés qui empruntent le canal. La conservation de cette organisation dans la région de constriction du canal (Figures 2 et 3 ) est un élément clé dans la fonction du pore, comme l'ont rapporté différents travaux sur le résidu Gly119. La substitution par un résidu Asp ou Glu se traduit par un encombrement croissant de la lumière de la porine qui perturbe la diffusion des antibiotiques et altère les propriétés du canal [22, 23]. De manière similaire, la substitution de résidus chargés, Aspl13 ou Aspl21 par Ala, entraîne une modification drastique des propriétés du pore et perturbe l'action de certaines molécules utilisant le canal [28]. Cette portion du canal joue ainsi un rôle essentiel dans la structure fonctionnelle, la disposition des charges, et le champ électrostatique localisé dans la constriction, et donc dans les interactions électrostatiques entre le soluté et la porine.

\section{Porines et résistance aux antibiotiques}

La résistance bactérienne aux antibiotiques est un problème majeur de santé publique. Les différents processus de résistance peuvent être regroupés en trois grands mécanismes qui concernent la modification: (1) de la cible (mutation, variabilité); (2) de l'antibiotique (dégradation, altération); et (3) de la concentration intracellulaire de la molécule (pénétration, systèmes d'efflux). La perméabilité membranaire intervient dans 
le contrôle de la concentration de différentes classes d'antibiotiques comme les $\beta$-lactamines ${ }^{1}$ ou les quinolones $^{2}$ via l'expression des porines ou des transporteurs-pompes [1-3].

Plusieurs résultats ont montré la relation existant entre la porine et la sensibilité aux $\beta$-lactamines et aux fluoroquinolones. Les analyses d'isolats résistants aux antibiotiques (Klebsiella, Enterobacter, Proteus, Pseudomonas ou Serratia) ont établi que la disparition des porines, associée à la production d'enzymes bactériennes détruisant le cycle $\beta$-lactame ( $\beta$-lactamases, céphalosporinases), est un élément clé dans la résistance aux $\beta$-lactamines. Chez Pseudomonas aeruginosa, la disparition d'une porine a été rapportée dans le cas de résistance à l'imipénème ${ }^{3}$ [25].

\section{Mutations, activité porine et résistance}

Des isolats d'entérobactéries synthétisant des quantités normales de porines mais montrant un profil de résistance similaire à un phénotype d'imperméabilité ont été décrits. La première identification d'un isolat d' $\varepsilon$. aerogenes de ce type, en 1998, a permis d'établir les bases moléculaires de ce mécanisme de résistance original: le remplacement d'un résidu Gly par un résidu Asp dans la boucle L3 (Figures 2 et 3), induisant le rétrécissement du canal et l'altération du champ électrostatique [29]. Des observations similaires ont été faites chez Neisseria gonorrhoeae [30] avec des mutations localisées dans l'équivalent de la boucle 3 des porines d' $\varepsilon$. coli. Dans ce cas, l'expression de pompes d'efflux ${ }^{4}$ augmente encore le niveau de résistance en assurant le transport et la libération des antibiotiques vers le milieu externe. Par ailleurs, il est important de mentionner l'existence d'un résidu Tyr supplémentaire dans la boucle $\mathrm{L3}$ de $0 \mathrm{mpK} 37$ de K. pneumoniae: la chaîne latérale diminue la taille du canal et bloquerait l'entrée des $\beta$ lactamines [31]. Ainsi, la bactérie exprimerait une porine naturellement inefficace pour la diffusion de certains antibiotiques. Ces observations posent le problème de la sélection possible de souches produisant des porines mutées ou adaptées sous une pression antibiotique.

${ }^{1}$ Les $\beta$-lactamines représentent une grande famille d'antibiotiques qui ont en commun une même structure chimique, le cycle $\beta$-lactame. Cette famille se partage en plusieurs groupes: les pénames (pénicillines), les céphèmes (céphalosporines), les carbapénèmes, les monobactames.

${ }^{2}$ Les quinolones sont des antibiotiques dérivant de la quinoléine. Les fluoroquinolones sont des quinolones comportant un atome de fluor.

${ }^{3}$ L'imipénème appartient au groupe des carbapénèmes, antibiotiques très résistants aux $\beta$-lactamases.

${ }^{4}$ Complexes protéiques présents dans l'enveloppe bactérienne impliqués dans l'expulsion des antibiotiques.

\section{Expression des porines et résistance}

Une des principales questions concerne le mécanisme impliqué dans la disparition des porines dans les souches résistantes. Récemment, la régulation rapide de l'expression de la porine d'E. aerogenes a été montrée chez un patient traité par l'imipénème. La prise de cet antibiotique s'accompagne de la disparition de la porine associée à la résistance de la souche aux $\beta$-lactamines, alors que l'arrêt du traitement entraîne sa réapparition chez la bactérie, redevenue sensible [37]. Dans plusieurs bactéries, la disparition des porines fait intervenir l'opéron mar, qui, via le petit ARN antisens micF, déstabilise l'ARNm de la porine et ce, conjointement à l'expression des pompes d'efflux [38-39]. Le système d'osmorégulation à deux composants ompR-envZ, qui règle l'expression de $0 \mathrm{mpC}-0 \mathrm{mpF}$ chez $\varepsilon$. coli [40], pourrait également jouer un rôle dans des isolats résistants. Par

\begin{tabular}{|c|c|c|c|}
\hline Bactérie & Protéine & $\begin{array}{l}\text { Résolution } \\
\text { (Angström) }\end{array}$ & Référence \\
\hline Comamonas acidovorans & $0 \mathrm{mp} 32$ & 2,1 & {$[6]$} \\
\hline Escherichia coli & $0 \mathrm{mpF}$ & 2,4 & [7] \\
\hline Escherichia coli & PhoE & 3 & [7] \\
\hline Klebsiella pneumoniae & $0 \mathrm{mpK36}$ & 3,2 & [8] \\
\hline Rhodobacter capsulatus & porine & 1,8 & [9] \\
\hline $\begin{array}{l}\text { Rhodopseudomonas } \\
\text { blastica }\end{array}$ & porine & 2 & {$[10]$} \\
\hline
\end{tabular}

Tableau I. Porines non spécifiques dont la structure tridimensionnelle est disponible.

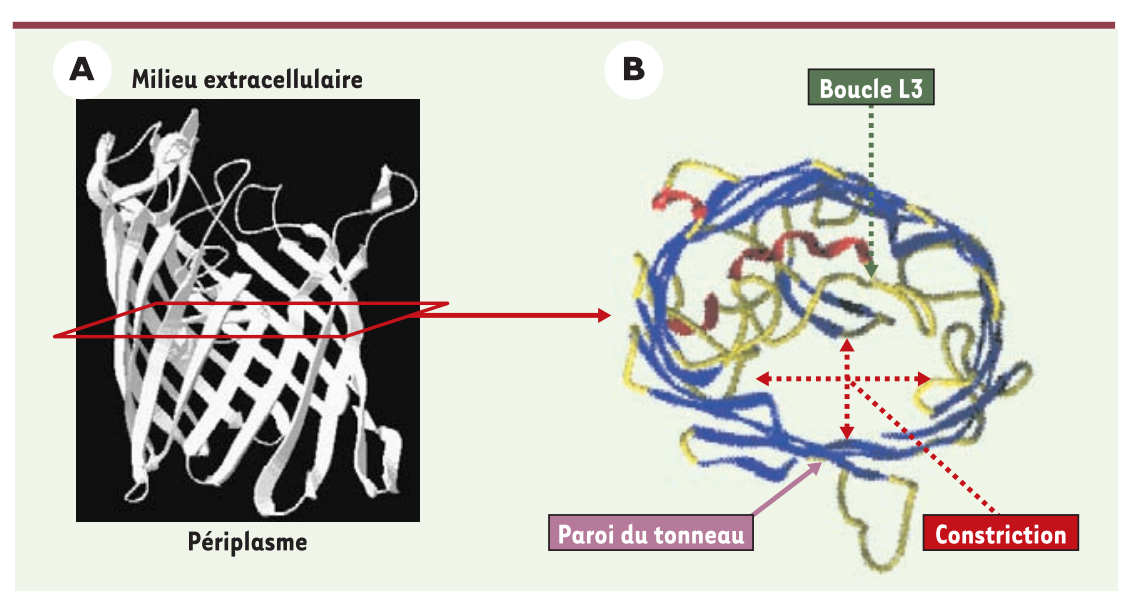

Figure 2. Structure de la porine $0 \mathrm{mpF}$ et de la région de constriction du canal. $A$. La porine $0 \mathrm{mpF}$ est une protéine dont la partie transmembranaire est constituée par des feuillets $\beta$. Les 16 feuillets $\beta$ antiparallèles définissent le tonneau $\beta$ contenant la région de constriction [7]. $\boldsymbol{B}$. Coupe transversale réalisée au niveau de la région de constriction de $0 \mathrm{mpF}$ formée par la boucle L3 infléchie dans le canal. 
ailleurs, l'expression de la protéine $0 \mathrm{mpX}$ diminue la synthèse des porines chez $\varepsilon$. aerogenes, $\varepsilon$. cloacae et K. pneumoniae par un mécanisme inconnu à ce jour. D'autres processus faisant intervenir des cascades de régulation génétique (via soxS, $\operatorname{ram} A, \mathrm{IHF}$ ) sont aussi associés à la diminution des porines. À côté de ces régulations génétiques possibles, l'influence de facteurs structuraux associés, comme celle du LPS dans l'assemblage, le transport et l'insertion dans la membrane externe, ainsi que celle des protéines chaperons, peuvent avoir un effet déterminant sur la quantité de porines exprimées, comme dans certaines souches leaky. Des facteurs externes ( $\mathrm{pH}$, osmolarité, sources de carbone, concentration en $\mathrm{O}_{2}$ ) peuvent aussi intervenir en fonction du site de colonisation ou du traitement, et induire la disparition de la porine [37-4l].

\section{Polyamines et résistance}

Les polyamines sont des molécules synthétisées par la bactérie dans des conditions particulières (stress, phase stationnaire de croissance). Ces petits composés portent des groupes aminés et peuvent contracter des interactions avec les acides aminés (Asp, Glu) localisés dans le canal de la porine. Différents travaux montrent la capacité de la spermine ou de la spermidine, de bloquer la diffusion des solutés et de la céphaloridine (céphalosporine de première génération) dans les porines d'E. coli $[34,35]$. La spermine restreint drastiquement la pénétration du céfépime (céphalosporine de dernière génération) et de la norfloxacine (fluoro-

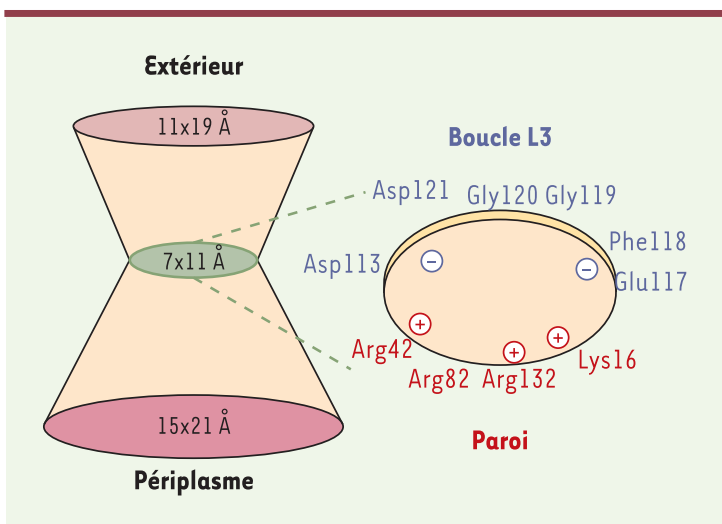

Figure 3. Représentation de la porine $0 \mathrm{mpF}$ et de la région de constriction. Les dimensions des différentes régions du canal, embouchure, constriction et delta périplasmique sont indiquées. Dans la partie droite, les acides aminés localisés dans la région de constriction et participant au champ électrostatique sont mentionnés : Aspl13 et Glul17 apportent une charge négative, alors que $\operatorname{Arg} 42, \operatorname{Arg} 132$, Lys 16 apportent une charge positive. quinolone) chez Enterobacter [19]. L'analyse de mutants de $0 \mathrm{mpF}$ a permis d'identifier deux positions au niveau de la constriction du canal, Aspll3 et 121, qui peuvent jouer le rôle de points d'ancrage pour la polyamine. Des mutants Asp113Ala et Asp121Ala sont en effet moins sensibles à l'effet de la spermine [28]. Les travaux de modélisation moléculaire ont par ailleurs identifié les acides aminés Aspl17 et Glu62 comme sites d'interaction possibles [42]. Ainsi la polyamine, par un glissement progressif dans le canal, serait responsable d'un encombrement transitoire $\mathrm{du}$ pore.

Cette hypothèse vient d'être argumentée par des résultats récents de notre laboratoire rapportant une inhibition de l'action de la colicine (qui utilise la porine $0 \mathrm{mpF}$ pour pénétrer dans la bactérie), lors de l'ajout simultané de spermine ou d'antibiotique (céfépime) [43]. On peut ainsi évaluer les affinités et les interactions en mettant en compétition les différents ligands (colicines, polyamines, antibiotiques) utilisant la porine. Des travaux préliminaires permettent également d'identifier des résidus impliqués dans le passage des polyamines, des $\beta$-lactamines et des bactériocines comme la colicine, montrant l'intérêt de cette approche.

\section{Modélisation moléculaire, étude dynamique et comparative de la diffusion}

Les travaux engagés par les équipes de A. Delcour, T. Schirmer, J. Tommassen, et par notre laboratoire, montrent que plusieurs résidus interviennent dans I'orientation et la diffusion des céphalosporines dans la lumière de la porine [22-24, 32-35]. À partir des données structurales disponibles sur les porines, et en positionnant l'ampicilline dans la constriction du canal OmpF, l'équipe de E.K. Nestorowich [36] a proposé que certains acides aminés (groupe «Arg42, 82 et 132 », ainsi que Glu117) jouent un rôle lors de la pénétration de l'antibiotique dans le canal. Nous avons caractérisé deux résidus importants pour le passage des céphalosporines dans le pore, Lys 16 et $\operatorname{Arg} 132$ [22, 24]. La substitution de ces résidus a un effet remarquable sur la diffusion des céphalosporines et des solutés. La localisation des charges sur la surface de la molécule d'antibiotique est maintenant un paramètre du dialogue électrostatique entre les deux partenaires, antibiotique et porine. Néanmoins le prépositionnement orienté de l'antibiotique utilisé par E.K. Nestorowich et al. [36] peut entraîner un masquage ou une amplification du rôle de résidus internes dans l'interaction intervenant dans la région de constriction de la porine. La description de souches produisant des porines modifiées pose 
clairement le problème de la réactivité potentielle de la molécule d'antibiotique et des acides aminés situés dans la zone de constriction du pore. Ces interactions, de faible énergie, avec différentes chaînes latérales, doivent être transitoires au cours de la diffusion afin de ne pas pérenniser la présence du soluté dans le canal et de ne pas induire des contraintes stériques altérant le passage de l'antibiotique.

À l'exception de récents zwitterions (céfépime, cefpirome, imipénème), la structure des $\beta$-lactamines prenait surtout en compte les notions d'affinité de l'antibiotique pour la cible bactérienne et de stabilité vis-à-vis des enzymes de résistance ( $\beta$-lactamases, céphalosporinases). Les travaux récents ouvrent la voie à une approche intégrant des paramètres importants dans la diffusion des antibiotiques permettant de faire progresser encore la conception et l'élaboration de nouvelles molécules.

\section{Conclusions}

Nous avons pu observer successivement l'émergence de bactéries montrant une expression réduite de porine, une absence de porine et, récemment, une porine mutée. Il a été conjointement montré que la synthèse des porines dans des souches colonisant un patient était en rapport avec l'antibiothérapie utilisée. Ainsi, le contrôle de la perméabilité fait intervenir diverses régulations qui dépendent du site de colonisation, du traitement et de la bactérie elle-même. Le problème clé est la rapidité d'adaptation de la bactérie en présence de l'antibiotique. La mise au point de sondes génétiques et de systèmes de quantification permettront de rechercher l'existence d'un contrôle pré- ou posttranscriptionnel dans ces souches et de suivre ainsi leur évolution et la cascade de régulation impliquée.

Les composants membranaires, comme le LPS et les systèmes d'adressage, participent également à l'intégrité et à l'assemblage fonctionnel des porines. La structure de la membrane peut être modifiée par des molécules ayant une activité membranotrope (peptides antimicrobiens, détergents ou antiseptiques). Il faut mentionner la synergie possible avec d'autres systèmes de régulation induits par des facteurs de l'environnement (salicylate, flavonoïdes) et avec certains composés (bactériocines) produits par la flore voisine. Des molécules peuvent être responsables d'un encombrement des porines et créer une imperméabilité par compétition. La synthèse de ces molécules peut intervenir dans un site de colonisation et entraîner une résistance efficace. Enfin, les systèmes d'efflux participent également à l'adaptation de la perméabilité membranaire aux conditions de l'environnement et à la réponse bactérienne à l'antibiothérapie. Ces divers éléments sont particulièrement importants car, même dans un cadre d'utilisation raisonnée des antibiotiques, on ne peut pas écarter une évolution de la résistance induite par d'autres facteurs ou d'autres molécules présentes dans le milieu. $\diamond$

\section{REMERCIEMENTS}

Je remercie J. Bredin et $A$. Favard pour leur aide à la rédaction du manuscrit.

\section{SUMMARY}

Bacterial porin and antibiotic susceptibility

In Gram negative bacteria, hydrophilic antibiotics such as $\beta$-lactams and fluoroquinolons used the bacterial porin channel during their entry. The balance of the porin expression level and the molecular parameters which govern the molecule diffusion through the pore are important physiological points. Acquired in vivo $\beta$-lactam resistance is often associated with porin loss, and recently clinical resistant strains synthetizing mutated porin have been described. These data highlight both the importance of the channel characteristics and the aminoacid residues involved in the drug diffusion process. In addition, several mechanisms, including various repressors or activators as well as molecules inhibiting the pore synthesis or activity, argue for the complexity and plasticity of the bacterial control of porin function. All these aspects play a key role in both membrane permeability and efficiency of the antibiotic resistance process. $\Delta$

\section{RéFÉRENCES}

1. Nikaido H. Outer membrane. In: Neidhardt FC, ed. Escherichia coli and Salmonella: cellular and molecular biology. Washington DC: ASM Press, 1996: 29-47.

2. Nikaido H. Prevention of drug access to bacterial targets: permeability barriers and active efflux. Science 1994; 264: 382-8.

3. Hancock REW. The bacterial outer membrane as a drug barrier. Trends Microbiol 1997; 5 : 37-42.

4. Koebnik R, Locher KP, Van Gelder P. Structure and function of bacterial outer membrane proteins: barrels in a nutshell. Mol Microbiol 2000; 37: 239-53.

5. Schirmer T. General and specific porins from bacterial outer membranes. J Struct Biol 1998; 121: 101-9.

6. Zeth K, Diederichs K, Welte W, Engelhardt H. Crystal structure of $0 \mathrm{mp} 32$, the anionselective porin from Comamonas acidovorans, in complex with a periplasmic peptide at $2.1 \AA$ resolution. Structure Fold Des 2000; 8: 981-92.

7. Cowan SW, Schirmer T, Rummel G, et al. Crystal structures explain functional properties of two E.coli porins. Nature 1992; 358: 727-33.

8. Dutzler R, Rummel G, Alberti S, et al. Crystal structure and functional characterization of $0 \mathrm{mpK} 36$, the osmoporin of Klebsiella pneumoniae. Structure Fold Des 1999; 7: 425-34.

9. Weiss MS, Kreusch A, Schiltz $\varepsilon$, et al. The structure of porin from Rhodobacter capsulatus at $1.8 \AA$ resolution. FEBS Lett 1991; $280: 379-82$. 


\section{RÉFÉRENCES}

10. Kreusch A, Neubüser A, Schiltz $\varepsilon$, et al. Structure of the membrane channel porin from Rhodopseudomonas blastica at $2.0 \AA$ resolution. Protein Sci 1994; 3: 58-63.

11. Zimmermann W, Rosselet A. Function of the outer membrane of Escherichia coli as a permeability barrier to beta-lactam antibiotics. Antimicrob Agents Chemother 1977; 12: 368-72.

12. Tokunaga M, Tokunaga $H$, Nakae T. The outer membrane permeability of Gram-negative bacteria. Determination of permeability rate in reconstituted membrane vesicles. FEBS Lett 1979; 106: 85-8.

13. Nikaido H, Rosenberg Ey. Porin channels in Escherichia coli. Studies with liposomes reconstituted from purified proteins. J Bacteriol 1983; 153: 241-52.

14. Montal M, Müller P. Formation of bimolecular membranes from lipid monolayers and study of their electrical properties. Proc Natl Acad Sci USA 1972; 69: 3561-6.

15. Schindler H, Rosenbusch JP. Matrix protein from Escherichia coli outer membranes forms voltage-controlled channels in lipid bilayers. Proc Natl Acad Sci USA 1978; 75 : 3751-5.

16. Delcour AH, Martinac B, Kung C, Adler J. A modified reconstitution method used in patch-clamp studies of Escherichia coli ion channels. Biophys J 1989; 56: 631-6.

17. DelaVega AL, Delcour AH. Cadaverine induces closing of $\varepsilon$. coli porins. EMBO J 1995; 14: 6058-65.

18. Iyer $\mathrm{R}$, Delcour AH. Complex inhibition of $0 \mathrm{mpF}$ and $0 \mathrm{mpC}$ bacterial porins by polyamines. J Biol Chem 1997; 272: 18595-601

19. Chevalier J, Mallea M, Pagès JM. Comparative aspects of the diffusion of norfloxacin, cefepime and spermine through the $F$ porin channel of Enterobacter cloacae. Biochem J 2000; 348: 223-7.

20. Saint N, Lou KL, Widmer C, et al. Structural and functional characterization of $0 \mathrm{mpF}$ porin mutants selected for larger pore size. Functional characterization. J Biol Chem 1996; 271: 20676-80.

21. Van Gelder P, Saint N, Phale P, et al. Voltage sensing in the PhoE and $0 \mathrm{mpF}$ outer membrane porins of Escherichia coli: role of charged residues. J Mol Biol 1997; 269: 468-72.

22. Simonet $V$, Malléa $M$, Pagès JM. Substitutions in the eyelet region disrupt cefepime diffusion through the Escherichia coli $0 \mathrm{mpF}$ channel. Antimicrob Agents Chemother 2000; 44 : 311-15.

23. Jeanteur D, Schirmer T, Fourel D, et al. Structural and functional alterations of a colicin resistant mutant of $0 \mathrm{mpF}$ from $\varepsilon$. coli. Proc Natl Acad Sci USA 1994; 91 : 10675-9.

24. Bredin J, Saint N, Malléa M, et al. Alteration of pore properties of Escherichia coli $0 \mathrm{mpF}$ induced by mutation of key residues in anti-loop3 region. Biochem J 2002; 363: 521-8.

25. Trias J, Nikaido H. Protein D2 channel of the Pseudomonas aeruginosa outer membrane has a binding site for basic aminoacids and peptides. J Biol Chem 1990; 265: 15680-4.

26. Karshikoff A, Spassov V, Cowan SA, et al. Electrostatic properties of two porin channels from Escherichia coli. J Mol Biol 1994; 240: 372-84.

27. Jeanteur D, Lakey JH, Pattus F. The bacterial porin superfamily: Sequence alignment and structure prediction. Mol Microbiol 1991; 5: 2153-64.

28. Iyer R, Wu Z, Woster PM, Delcour AH. Molecular basis for the polyamine-
OmpF porin interactions: inhibitor and mutant studies. J Mol Biol 2000; 297: 933-45.

29. Dé $\varepsilon$, Basle $A$, Jaquinod $M$, et al. A new mechanism of antibiotic resistance in Enterobacteriaceae induced by a structural modification of the major porin. Mol Microbiol 2001; 41 : 189-98.

30. Veal WL, Nicholas RA, Shafer WM. Overexpression of the MtrC-MtrD-MtrE efflux pump due to an mtrR mutation is required for chromosomally mediated penicillin resistance in Neisseria gonorrhoeae. J Bacteriol 2002; 184: 5619-24.

31. Domenech-Sanchez A, Hernandez-Alles S, Martinez-Martinez L, et al. Identification and characterization of a new porin gene of Klebsiella pneumoniae: its role in $\beta$-lactam antibiotic resistance. J Bacteriol 1999; 181: 2726-32.

32. Phale PS, Schirmer T, Prilipov A, et al. Voltage gating of Escherichia coli porin channels: role of the constriction loop. Proc Natl Acad Sci USA 1997; $94: 6741-5$.

33. Eppens EF, Saint N, van Gelder P, et al. Role of the constriction loop in the gating of outer membrane porin PhoE of Escherichia coli. FEBS Lett 1997; 415: 317-20.

34. DelaVega AL, Delcour AH. Polyamines decrease Escherichia coli outer membrane permeability. J Bacteriol 1996; 178: 3715-21.

35. Samartzidou H, Delcour AH. Excretion of endogenous cadaverine leads to a decrease in porin-mediated outer membrane permeability. J Bacteriol 1999; 181: 791-8.

36. Nestorovich $\varepsilon$ K, Danelon C, Winterhalter M, Bezrukov SM. Designed to penetrate: time-resolved interaction of single antibiotic molecules with bacterial pores. Proc Natl Acad Sci USA 2002; 99: 9789-94.

37. Bornet C, Davin-Regli A, Bosi C, et al. Imipenem resistance of Enterobacter aerogenes mediated by outer membrane impermeability. J Clin Microbiol 2000; 38: 1048-52.

38. Delihas N, Frost S. MicF: an antisense RNA gene involved in response of Escherichia coli to global stress factors. J Mol Biol 2001; 313: 1-12.

39. Aleskun MN, Levy SB. The mar regulon: multiple resistance to antibiotics and other toxic chemicals. Trends Microbiol 1999; 7: 410-3.

40. Pratt LA, Hsing W, Gibson KE, Silhavy TJ. From acids to osmZ: multiple factors influence synthesis of $0 \mathrm{mpF}$ and $0 \mathrm{mpC}$ porins in Escherichia coli. Mol Microbiol 1996; 20: 911-7.

41. Low AS, MacKenzie FM, Gould IM, Booth IR. Protected environments allow parallel evolution of a bacterial pathogen in a patient subjected to long-term antibiotic therapy. Mol Microbiol 2001; 42: 619-30.

42. Vidal S, Brouant P, Chevalier J, et al. Computer simulation of spermineporin channel interactions. In Vivo 2002; 16: 111-6.

43. Bredin J, Simonet V, lyer R, et al. Colicins, spermine and cephalosporins: a competitive interaction with the $0 \mathrm{mpF}$ eyelet. Biochem J 2003; 376: $245-52$ 